\title{
Single Microparticle Separation by Thermal Bubble Actuation in Microfluidic Chips
}

\author{
Kehsin Tseng and Chingfu Tsou* \\ Department of Automatic Control Engineering, Feng Chia University, \\ 100 Wenhwa Road, Seatwen, Taichung 40724, Taiwan, R.O.C.
}

(Received June 10, 2013; accepted October 2, 2013)

Key words: microparticle separation, thermal bubble, microfluidic chip

Cell and microparticle separation in microfluidic systems, which is typically achieved by applying forces to the targets to guide them along different paths, has recently gained significant attention for the preparation of samples in biological and chemical studies. Therefore, we propose a new microfluidic chip with a resistive silicon-based microheater, where noncontact force is applied by thermal bubble actuation for microparticle separation. The static and dynamic electrothermal coupling characteristics of the resistive microheater and the flow behaviors of fluid in the microchannel were evaluated by finite-element analysis and using the commercial simmulation software COMSOL, respectively, to provide an applicable design. The feasibility of a thermal bubblepumping fluid to manipulate a microbead of $20 \mu \mathrm{m}$ diameter has also been verified by experiments. It is expected that the proposed microfluidic chip can be applied to the separation of cells, such as live cells in amniotic fluid.

\section{Introduction}

During the last decade, the development of microfluidic chips has become an emerging topic because they offer benefits over conventional macroscale instruments, including lower cost, fewer samples and less reagent consumption, adaptability for automation, and the promise of portable point-of-care devices. In general, cell separation for bioassays broadly refers to the procedures undertaken to isolate or purify cells, biomolecules, or other targets of interest from specimen samples mainly for the acquisition or enrichment of targets, the removal of impurities or interfering constituents, the enhancement of product stability, or for sorting purposes. The basic sorting rationale is primarily based on distinctions in size, shape, density, and other physical or biochemical features. For example, to isolate cells in a suspension or to separate particular cell species from a cell pool, centrifugation techniques are commonly used.(1)

Recently, several microfluidic systems have been intensively explored for various cell-based applications, such as drug screening and testing, ${ }^{(2,3)}$ cell physiology, ${ }^{(4)}$ and

${ }^{*}$ Corresponding author: e-mail: cftsou@fcu.deu.tw 
cancer cell metastasis. ${ }^{(5)}$ It has been demonstrated that microscale cell separation or isolation can be realized by adopting different mechanisms, such as microchannels with specific geometric or structural designs, ${ }^{(6)}$ and by manipulating microparticles using noninertial forces, such as acoustic, magnet-based, ${ }^{(7)}$ and dielectrophoresis (DEP) forces. ${ }^{(8,9)}$ In addition, a number of micropumps with gas bubbles have recently been applied to microfluidic devices and employed to manipulate microparticles using noncontact forces, leading to numerous successful microchip designs for particle and cell separation. For example, a patch-clamp (PC) microchip with cell sorting and positioning functions has been reported and shows promise for use in an automated PC microsystem. ${ }^{(10)}$ On the basis of these methods, actuators have been regarded as a promising strategy for cell sorting and separation. ${ }^{(11,12)}$ They only require a few samples to accomplish bioanalysis and effectively shorten the cycle time. However, the separation or isolation of cells from a fresh specimen, in such microscale operations, still has technical challenges, such as a complex fabrication process and low efficiency results.

To solve the problems mentioned above, we present a thermal bubble-actuated microfluidic-chip-integrated screening element for microparticle separation that is based on a simple process with a silicon-on-isolator (SOI) wafer. A resistive bulk microheater with vertical sidewalls is utilized to generate reliable thermal bubbles, thereby implementing the functions of a micropump for manipulating microparticles. A microheater set at a retreated position from the main channel was designed to minimize the thermal effect. The detailed performance of these components has been investigated by experiments and discussion in this study. The proposed microfluidic chip can be applied to cell sorting, such as with a bubble-jet cell sorter, and to amniotic cell screening.

\section{Design and Simulation}

\subsection{Design concept}

The schematic view of the proposed microfluidic chip for microparticle separation is shown in Fig. 1. The key components include a screening element with multiple through-holes and a microfluidic chip with microchannel routes, reservoirs, and arch-

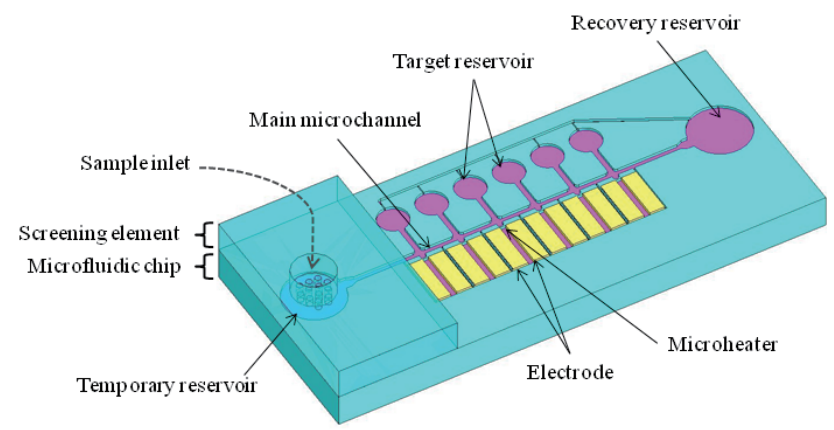

Fig. 1. (Color online) Schematic view of a microfluidic chip with thermal bubble micropump for microparticle separation. 
type microheaters, which can be fabricated by silicon bulk micromachining. The reservoir below the screening element is used to provide a temporary storage for the fluid dropped from the sample inlet. Thus, when the fluid with different sizes of microparticles is injected into the sample inlet, specific microparticles, with sizes smaller than the through-hole, could drop into the temporary reservoir and then move to the main microchannel. Once the microparticles move close to the microheater, a dynamic thermal bubble, which results from alternating voltage applied to the electrodes, is generated quickly and used as a micropump to achieve fluid transportation. At the same time, the target microparticle following the disturbed fluid could be moved into the target reservoir for inspection. When the microheater is turned off, the fluid will flow into the recovery reservoir along the main microchannel.

Detailed configurations of the screening structure and microheater are shown in Figs. 2(a) and 2(b), respectively. Figure 2(a) shows the cross section of the screening structure. The upper infusion cavity and bottom multi-through-holes are used to contain the fluid sample and as a size filter, respectively. Therefore, the smaller microparticles can pass through the through-hole to a temporary reservoir and the larger microparticles remain in the tank, achieving the collection of the target microparticles. Infusion tanks of specific sizes can be easily fabricated by silicon anisotropic wet etching. In addition, inductively coupled plasma (ICP) anisotropic dry etching is selected to fabricate the through-holes, since its minimum size is dependent on the aspect ratio in deep silicon etching. The structure of the microheater in Fig. 2(b) consists of a single-crystal silicon base layer, a silicon dioxide $\left(\mathrm{SiO}_{2}\right)$ isolation layer and a structural layer with a resistive arch-type microheater and a main microchannel made from a single crystal of silicon. Thus, when applying voltage on the electrodes $(+\mathrm{V}$ and $-\mathrm{V})$, the microheater temperature will quickly increase at the neck portion with an increase in the applied voltage. Once the temperature reaches the fluid's critical phase change condition, bubbles that act as a piston to displace fluid away from itself are nucleated. It is noted that the microheater is set away from the main microchannel at a specified distance $(L)$, so as to avoid the effect of high temperature on the sample. Further details of the design and performance evaluation of the microheater have been discussed in our previous study.(13)

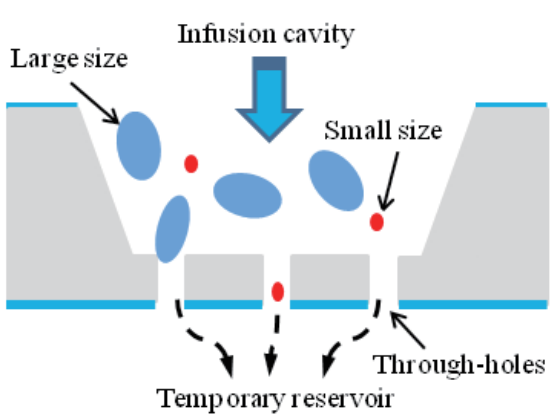

(a)

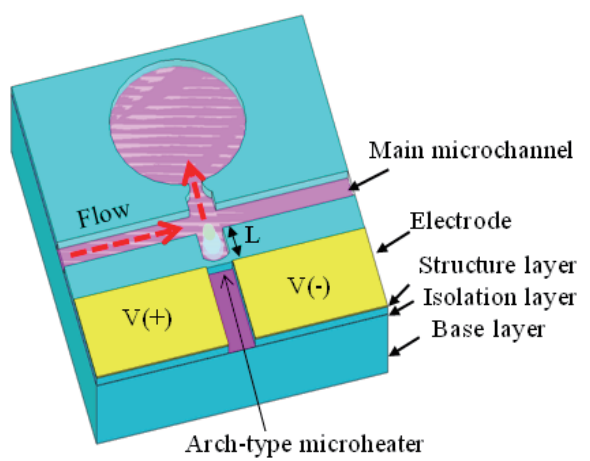

(b)

Fig. 2. (Color online) Schematic view of (a) a screening element and (b) a silicon-based vertical microheater. 


\subsection{Microheater and flow analysis}

In an effort to understand the temperature distribution on the microheater, a finiteelement model for the heater shown in Fig. 2(b) was established using commercial ANSYS software. To satisfy the realistic specifications of the SOI-MEMS process described in this paper, both base and structural layers are assumed to be single-crystal silicon, and the intermediate isolation layer is $\mathrm{SiO}_{2}$. The thickness of the structure layer and the width of the fluidic microchannel were both set at $70 \mu \mathrm{m}$, and the preliminary dimensions of the microheater were designed to be 100,40 , and $20 \mu \mathrm{m}$ for the length, boundary width, and neck width, respectively. In addition, three distances L of 100, 200, and $300 \mu \mathrm{m}$ were also designed to evaluate the thermal effect on microparticles. Note that the dimensions of the full models were set at $1.1 \times 1.5 \times 0.5 \mathrm{~mm}^{3}$ to reduce the effects of thermal conductivity. The linearly temperature-dependent material properties of each component used for simulation are shown in Table 1.

According to previous experimental results, thermal bubbles collapse at an applied voltage greater than $12 \mathrm{~V}$. On the basis of this condition, a square-wave AC voltage of $12.5 \mathrm{~V}$ was applied to the microheater model to realize the thermal effect. The simulation results of temperature distribution for the microheater with water contained in a microchannel are shown in Figs. 3(a)-3(c). Figure 3(a) shows the highest temperature, $720{ }^{\circ} \mathrm{C}$, at the neck portion of the microheater, when the distance $L$ is $100 \mu \mathrm{m}$. Also, the temperature close to the main microchannel is around $120{ }^{\circ} \mathrm{C}$. This temperature might affect the property of a microparticle, especially a living cell. However, in cases where the distance $L$ is large, $200 \mu \mathrm{m}$, the temperature of the main microchannel can be reduced to less than $35^{\circ} \mathrm{C}$, as shown in Figs. 2(b) and 2(c). This result reveals that selecting an appropriate distance between the microheater and main microchannel can effectively reduce the thermal effect. Thus, a high-performance microheater can be effectively designed for microfluidic chip applications.

The commercial simulation software COMSOL was also used to analyze the flow behaviors in thermal bubble pumping of fluid. On the basis of the layout of the microchannel in Fig. 2(b), the model is established and shown in Fig. 4(a). The length and width of the main microchannel were designed to be 1 and $0.1 \mathrm{~mm}$, respectively. The distance between the microheater and main microchannel was designed to be 100 $\mu \mathrm{m}$, and the bottom and upper necking widths of the vertical microchannel were 100 and $50 \mu \mathrm{m}$, respectively. The liquid in the microchannel is assumed to be water with a laminar flow. For the above conditions, the simulation results of flow behavior are shown in Fig. 4(b), where the pressure differential between the inlet and outlet is 100 MPa. This figure demonstrates that the liquid mostly flows to the outlet, except for the boundary layer of the fluid. However, when a pressure of $150 \mathrm{MPa}$ is applied to the

Table 1

Linear and temperature-dependent material properties of each component in electrothermal coupling simulation.

\begin{tabular}{lcccc}
\hline Material & $D\left(\mathrm{~kg} / \mathrm{m}^{3}\right)$ & $K(\mathrm{w} / \mathrm{m} \cdot \mathrm{k})$ & $C(\mathrm{~J} \cdot \mathrm{k} / \mathrm{kg})$ & $R(\mathrm{~W} \cdot \mathrm{m})$ \\
\hline $\mathrm{Si}$ & 2300 & 157 & 700 & 0.0002 \\
$\mathrm{SiO}_{2}$ & 2200 & 1.1 & 670 & $1 \times 10^{14}$ \\
$\mathrm{Al}$ & 2700 & 236 & 900 & $0.7 \times 10^{-8}$ \\
Water & 1000 & 0.7 & 4180 & 20 \\
\hline
\end{tabular}




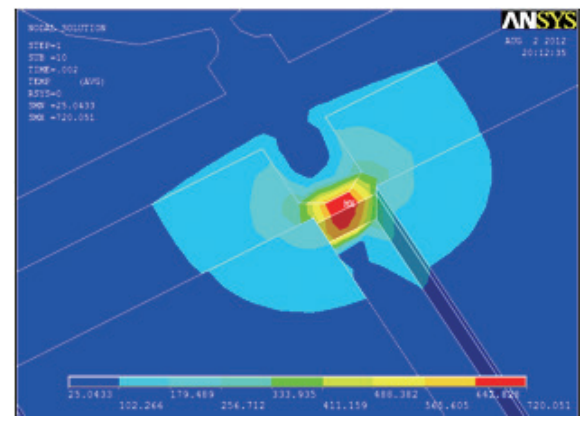

(a)

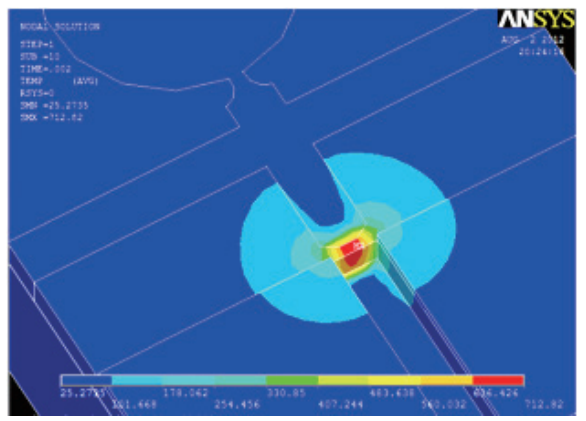

(b)

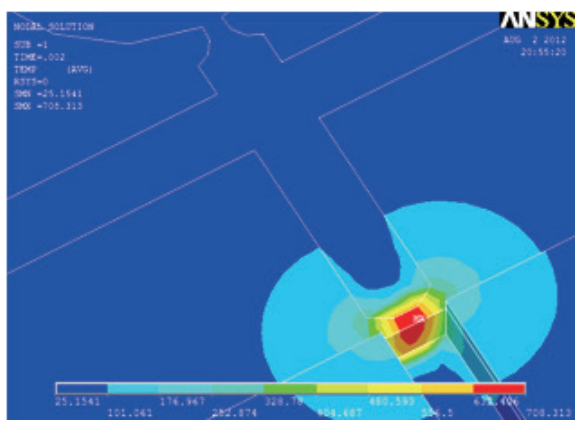

(c)

Fig. 3. (Color online) Simulation results of temperature distribution of the microheater with applied voltage of $12.5 \mathrm{~V}$ : (a) $L=100 \mu \mathrm{m}$, (b) $L=200 \mu \mathrm{m}$, and (c) $L=300 \mu \mathrm{m}$.

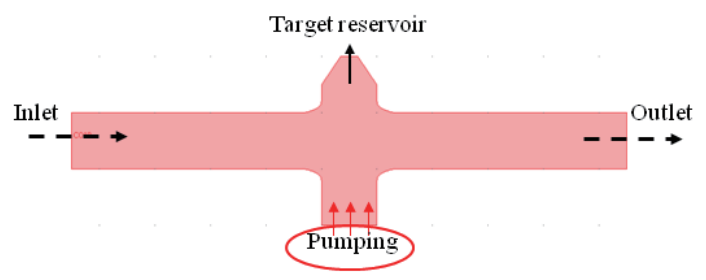

(a)

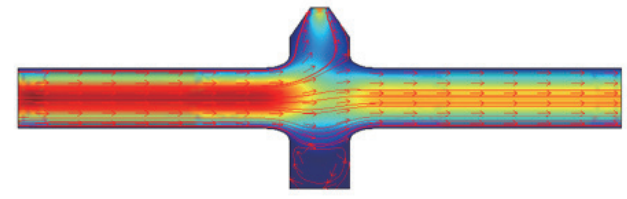

(b)

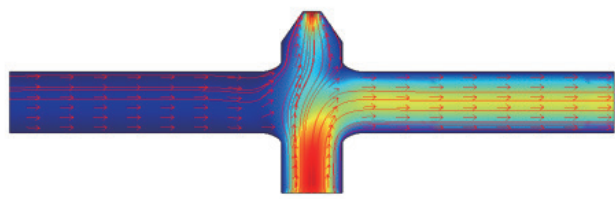

(c)

Fig. 4. (Color online) (a) Established model for fluidic analysis and simulation results of flow behavior, under a microchannel (b) without and (c) with bubble actuation.

pumping section, almost all the liquid in the main microchannel is forced to flow to the target reservoir. Thus, an active micropump with thermal bubbles can satisfy the pumping requirement for microparticle manipulation. 


\section{Experiment and Discussion}

\subsection{Fabrication}

For the screening element, the fabrication process consists of two photolithographic masks: one defines the etching window for the infusion cavity and the other is used to pattern a micro-through-hole. Details of the fabrication process are shown in Fig. 5. The process begins with the silicon nitride being deposited on a double-sided silicon wafer [Fig. 5(a)]. The photolithographic process (mask \#1) and deep reactive ion etching (DRIE) dry etching, as well as $\mathrm{KOH}$ wet etching, are used in turn to fabricate the infusion cavity [Figs. $5(\mathrm{~b})-5(\mathrm{~d})]$. Wafer bonding and the patterning of the etching window of a micro-throughhole are then implemented by photoresist (PR) adhesion and the photolithography process (mask \#2), respectively [Fig. 5(e)]. After that, the micro-through-holes are fabricated by ICP dry etching [Fig. 5(f)]. Finally, the bottom wafer and PR are removed to complete the screening element [Fig. $5(\mathrm{~g})]$. Figure 6 shows the fabricated siliconbased screening structure with an infusion cavity and multi-through-holes. The thickness of the silicon substrate is around $525 \mu \mathrm{m}$. The infusion cavity was etched in $\mathrm{KOH}(20 \%)$ at $72{ }^{\circ} \mathrm{C}$ for $7 \mathrm{~h}$, resulting in the $1.6 \times 1.6 \mathrm{~mm}^{2}$ etching window and an etching depth of around $420 \mu \mathrm{m}$. In addition, the diameter of each micro-through-hole was $50 \mu \mathrm{m}$ for the array units with a pitch of $100 \mu \mathrm{m}$, as seen in the top-view image shown in Fig. 5(b).

The detailed fabrication process of the microfluidic chip is shown in Fig. 7. The process begins with a highly doped SOI wafer with a structure layer of $70 \mu \mathrm{m}$ buried in a $2-\mu \mathrm{m}$-thick oxide layer [Fig. 7(a)]. The resistance of the structure layer is about 0.0002 $\Omega \cdot \mathrm{m}$. First, a $1-\mu \mathrm{m}$-thick aluminum layer is deposited by evaporation and patterned on the structural layer to form connecting pads [Fig. 7(b)]. Then, the structures of the

(a) Silicon nitride deposition

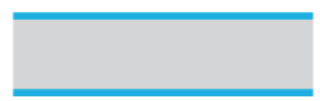

(b) Etching of window pattern

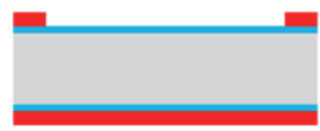

(c) RIE dry etching

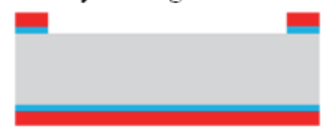

(d) $\mathrm{KOH}$ wet etching

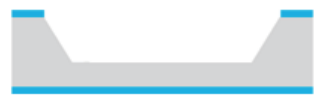

(e) Wafer bonding and etching of window pattern

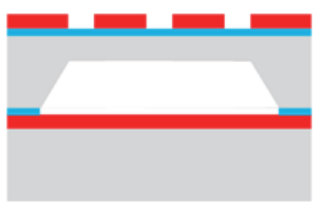

(f) ICP anisotropic etching

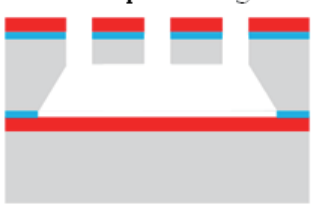

(g) Wafer andPR removal

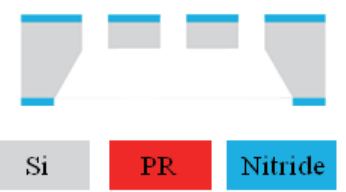

Fig. 5. (Color online) Detailed process flow for fabricating the screening structure with infusion cavity and through-hole. 


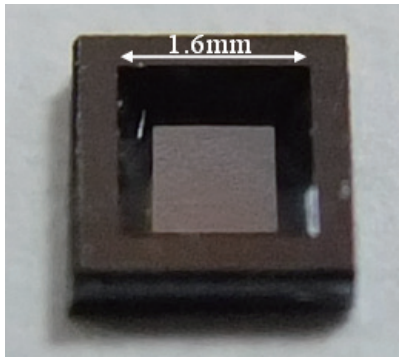

(a)

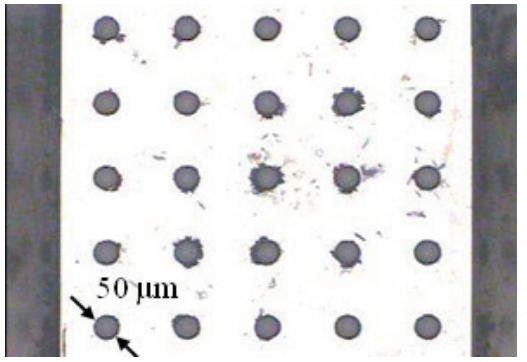

(b)

Fig. 6. (Color online) Typical fabrication results for the screening structure: (a) three-dimensional view and (b) top view of micro-through-holes.

(a) SOIwafer

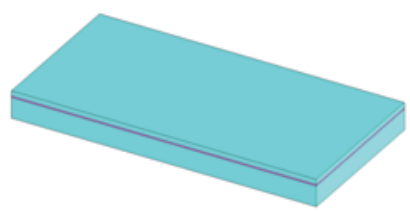

(b) Electrode patterning

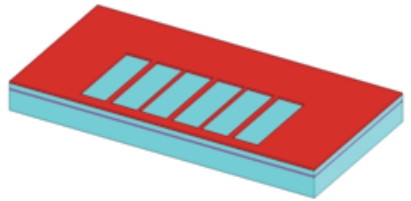

(c) Al deposition and lift-off

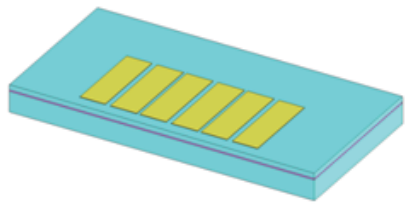

(d) Etching of window pattern

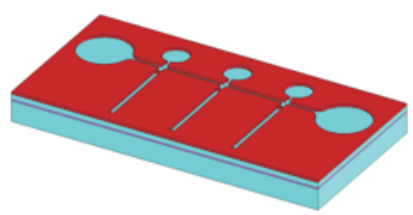

(e) ICP etching

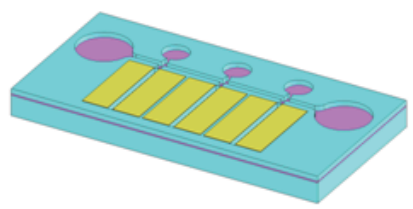

\begin{tabular}{l|l|l|l|} 
Si & Oxide & Al & PR
\end{tabular}

Fig. 7. (Color online) Detailed process flow for fabricating the microfluidic chip with microheater and microchannel.

microheater and microchannel are fabricated by photolithography patterning [mask \#1] and deep silicon dry etching, respectively [Figs. 7(c) and 7(d)]. Finally, a thin glass plate is bonded on the wafer to complete the microfluidic chip [Fig. 7(e)]. A typical fabrication result for the microfluidic chip of $1.4 \times 4.2 \mathrm{~cm}^{2}$ size is shown in Fig. 8. Three microheaters and six electrodes at the chip center are used as micropumps and for electrical input, respectively. The main microchannel is used to guide the flow direction of the microfluidic chip and connect the temporary reservoir, target reservoir, and collecting reservoir. The lower images in Fig. 8 show the representative diagram of the arch-type microheater region and its feature sizes. In this sample, the measured width of the microchannel is about $100 \mu \mathrm{m}$, and the length, boundary width, and neck width of the microheater are about 100, 40, and $20 \mu \mathrm{m}$, respectively. 


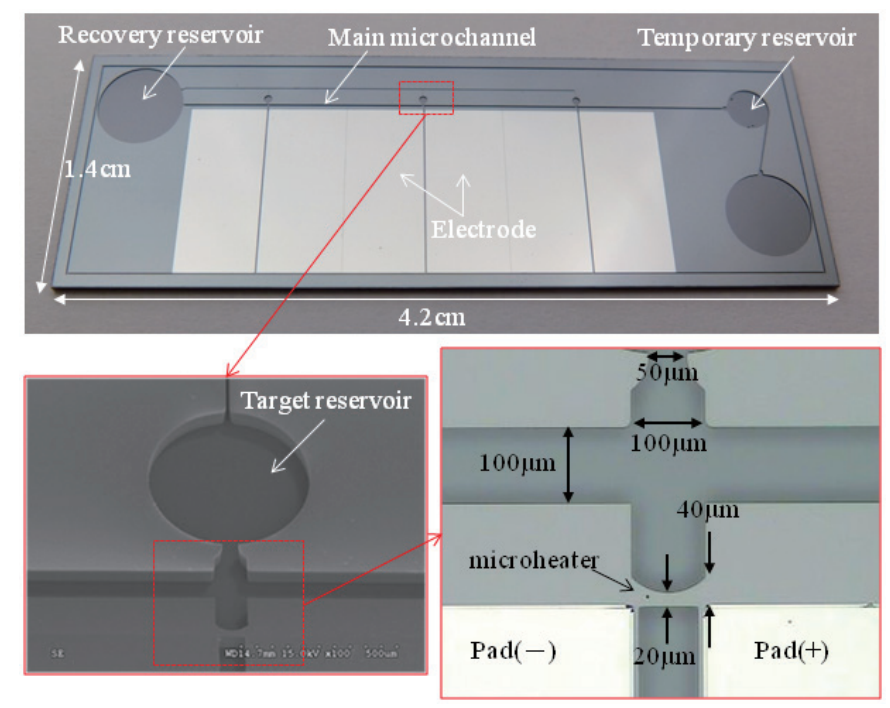

Fig. 8. (Color online) Typical fabrication results of the proposed microfluidic chip.

\subsection{Measurement}

To validate the feasibility of microparticles passing through the screening element, especially for cell separation, microbeads 20 and $60 \mu \mathrm{m}$ in diameter were mixed with water at the specific ratio of $0.1: 1: 10$. Then, the fluid was injected into the upper cavity of the screening structure without any stirring. Note that the properties of the selected microbeads can represent amniotic fluid cells, where the sizes of living and dead cells are about $25 \mu \mathrm{m}$ and larger than $50 \mu \mathrm{m}$, respectively. ${ }^{(14)}$ Figure 9 shows the typical separation results of the microbeads. Figure 9(a) shows that large microbeads still remain in the cavity because of their large diameter, but several small microbeads remain at the bottom. As can be seen in Fig. 9(b), most of the microbeads with diameters of 20 $\mu \mathrm{m}$ could pass along the micro-through-hole and drop into the temporary reservoir of the microfluidic chip.

For the fabricated microfluidic chip shown in Fig. 8, the sizes of bubbles obtained under various DC voltages were measured using an optical microscope with a highresolution position gauge. The typical formation processes of thermal bubbles are shown in Fig. 10. When the input current level is low, i.e., the applied voltage is less than 5 $\mathrm{V}$, no bubbles are generated. When the voltage is increased to higher than $5 \mathrm{~V}$, bubble nucleation is induced owing to the liquid phase change. After that, bubbles grow rapidly with increasing voltage, as shown in Figs. 10(a)-10(d). The variation in bubble diameter with applied voltage is shown in Fig. 11. The bubbles showed the reverse trend when decreasing the applied voltage. While the applied voltage increased continuously, the bubbles expanded and finally collapsed at an applied voltage greater than $10 \mathrm{~V}$. Once the applied voltage was greater than $10 \mathrm{~V}$, thermal bubbles can regrow quickly, resulting in the cycle time of bubble oscillation with implosion being about $0.2 \mathrm{~s}$.

According to the above experimental results, a square wave AC voltage of $10.5 \mathrm{~V}$ with a frequency of $100 \mathrm{~Hz}$ and a duty cycle of $50 \%$ was applied to the microheater 


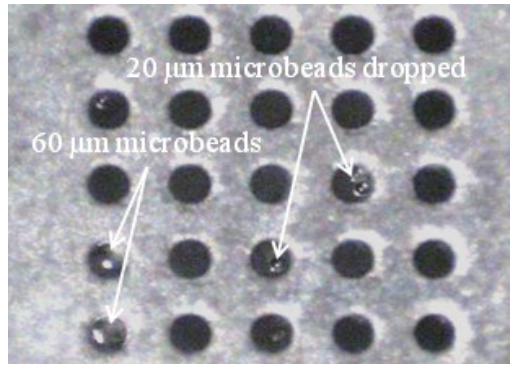

(a)

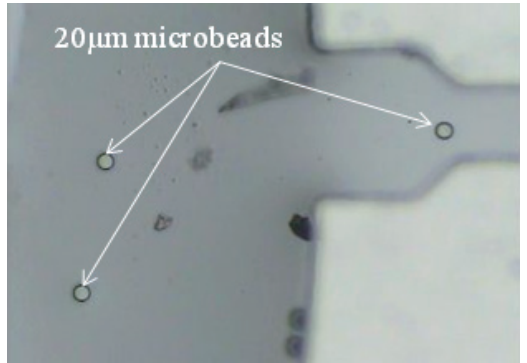

(b)

Fig. 9. Typical experimental results of microparticle separation using screening element.

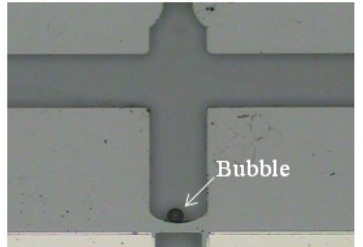

(a)

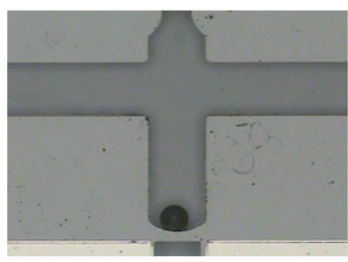

(c)

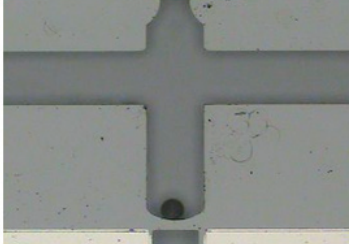

(b)

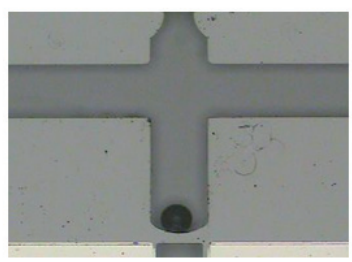

(d)

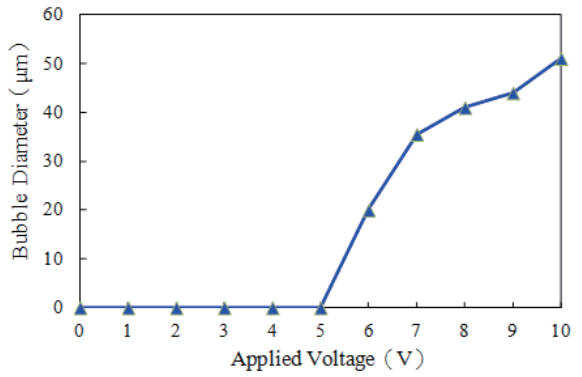

Fig. 10 (left). Variation of bubble formation with applied DC voltage: (a) $7 \mathrm{~V}$, (b) $8 \mathrm{~V}$, (c) $9 \mathrm{~V}$, and (d) $10 \mathrm{~V}$.

Fig. 11 (right). Variation of bubble size with applied voltage.

for single microparticle manipulation. First, when a microbead flowed into the main microchannel, it was observed with a microscope. Electrical power was applied to the microheater $200 \mu \mathrm{m}$ away from the microchannel. At this moment, with the time (t) set at zero, a dynamic thermal bubble was generated, forcing fluid to flow into the target reservoir, as shown in Fig. 12(a). During this experiment, the pressure drop between the inlet and outlet was controlled so that the fluid exhibited laminar flow in the main microchannel and the flow rates were stable at $1 \mathrm{~mm} / \mathrm{s}$. With the single microbead approaching the intersection of the microchannel, the fluid actuated by the thermal bubble carried the microbead into the target reservoir within a short time of $0.2 \mathrm{~s}$, as shown in Figs. 12(b)-12(c). Highly reproducible experimental results, similar to the three intercepted microbeads shown in Fig. 12, have confirmed the practicability of this microparticle separation technique. 


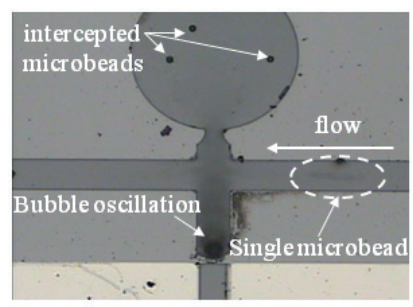

(a)

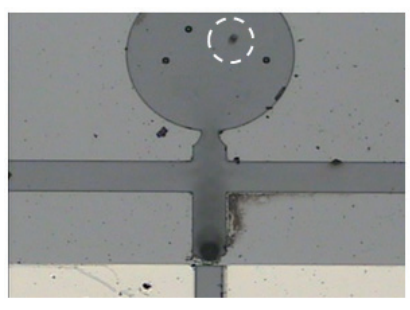

(c)

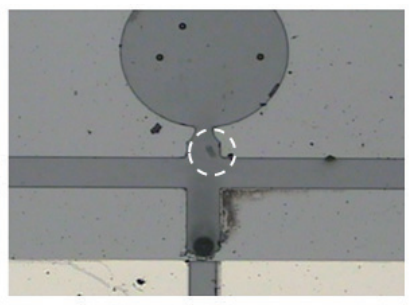

(b)

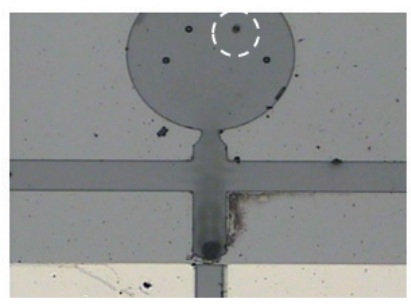

(d)

Fig. 12. Optical images of the manipulated microbeads under various actuation times: (a) $t=0 \mathrm{~s}$, (b) $t=0.2 \mathrm{~s}$, (c) $t=0.4 \mathrm{~s}$, and (d) $t=0.6 \mathrm{~s}$.

\section{Conclusions}

A microfluidic chip, with a silicon-based vertical microheater and microchannel routes based on an SOI wafer, has been successfully fabricated and demonstrated for microparticle separation. SOI-MEMS technology with a simple process has been implemented to realize the chip base with an arch-type resistive microheater and highaspect-ratio microchannel. The characteristics and practicability of the microheater as a micropump to manipulate microparticles were evaluated by simulation and experiment. A silicon-based substrate with a micro-through-hole of $50 \mu \mathrm{m}$ and a microheater with dimensions of $100 \times 40 \times 20 \mu \mathrm{m}^{3}$ were fabricated as the screening structure and micropump, respectively. Experimental results show that microparticles of a specific size can be sifted easily by the multi-through-holes of the screening device. The separation of a single microbead of $20 \mu \mathrm{m}$ diameter has been accomplished by the dynamic thermal bubble actuation. A series of operations for multifunctional tests also provides evidence that the flow manipulation was easily controlled, and the performance results show high reproducibility. These properties make the proposed microfluidic chip suitable for cell separation and the inspection of biological features. 


\section{Acknowledgements}

This project was supported by the National Science Council of Taiwan under grant NSC-100-2628-E-035-008. The authors thank the Precision Instrument Support Center of Feng Chia University, Instrument Technology Research Center (ITRC), the Nano Facility Center (NFC) of National Chiao Tung University, and the NSC National Nano Device Laboratory (NDL) for providing the fabrication facilities.

\section{References}

1 M. Yamada, K. Kano, Y. Tsuda, J. Kobayashi, M. Yamato, M. Seki and T. Okano: Biomed Microdevices 9 (2007) 637.

2 K. S. Yun, D. Lee, H. S. Kim and E. Yoon: Sens. Actuators B 143 (2009) 387.

3 H. W. Wu, X. Z. Lin, S. M. Hwang and G. B. Lee: Biomed Microdevices 11 (2009) 869.

4 Q. Tan, J. Chen, Y. Zheng, B. K. Chen and Y. Sun: J. Microelectromech. Syst. 25 (2012) 757.

5 Y. Fu, A. M. J. Vandongen, T. Bourouina, W. T. Park, M. Y. Je, J. M. Tsai, D. L. Kwong and A. Q. Liu: J. Microelectroech. Syst. 25 (2012) 773.

6 Y. Yamaguchi, T. Arakawa, N. Takeda, Y. Edagawa and S. Shoji: Sens. Actuators, B 136 (2009) 555.

7 T. Laurell, F. Petersson and A. Nilsson: Chem. Soc. Rev. 36 (2007) 492.

8 H. Park, D. Kim and K. S. Yun: Sens. Actuators, B 150 (2010) 167.

9 T. Kano, T. Inaba, Y. Gu and N. Miki: J. Microelectroech. Syst. 25 (2012) 745.

10 S. Penmetsa, K. Nagrajan, Z. Gong, D. Mills and L. Que: Solid-State Sensors, Actuators and Microsystems Conference 15 (2009) 81.

11 C. C. Chen, J. S. Wang and O. Solgaard: Sens Actuators, B 117 (2006) 523.

12 P. H. Chen, W. C. Chen and S.H. Chang: Int. J. Mech. Sci. 39 (1997) 683.

13 C. F. Tsou and C. H. Huang: J. Microelectromech. Syst. 18 (2009) 852.

14 D. Fauza: Best Pract. Res. Clin. Obstetrics Gynaecology 18 (2004) 877. 